\title{
A Non-Intrusive Method to Inferring Linear Port-Hamiltonian Realizations using Time-Domain Data
}

\author{
Karim Cherifi ${ }^{1}$, Pawan Goyal ${ }^{1}$ and Peter Benner ${ }^{1,2}$
}

\begin{abstract}
Port-Hamiltonian systems have gained a lot of attention in recent years due to their inherent valuable properties in modeling and control. In this paper, we are interested in constructing linear port-Hamiltonian systems from time-domain input-output data. We discuss a non-intrusive methodology that is comprised of two main ingredients - (a) inferring frequency response data from time-domain data, and (b) constructing an underlying port-Hamiltonian realization using the inferred frequency response data. The proposed method is illustrated by means of two numerical examples.
\end{abstract}

\section{INTRODUCTION}

In this paper, we focus on a non-intrusive way for the construction of a class of linear structured systems. Nonintrusive modeling has received a lot of attention recently due to its data-driven nature, see, e.g., [1], [2], [3], [4]. There are primarily two fundamental ways to obtain data, leading to data-driven modeling. The first is to experiment in a laboratory to obtain data. This approach is often desirable when very little knowledge is available about the process and parameters. In the second approach, one can simulate a process or model using proprietary software with desired parameters and conditions. Indeed, one can seek to obtain the underlying model in a matrix-vector form, however, it is a quite challenging task to extract the model, or sometimes even impossible due to intellectual property rights. Nevertheless, one can easily obtain simulated data using simulation software. Anyhow, in both cases, the goal is to create a model that describes the data and incorporates (if available) any additional knowledge such as conversational laws, and a particular desired properties such as port-Hamiltonian structure.

In this work, our focus lies on inferring linear timeinvariant (LTI) port-Hamiltonian $(\mathrm{pH})$ systems using timedomain input-output data. PH systems are structured representations of dynamical systems [5], [6], [7], [8], [9] that typically arise, e.g., from energy-based modeling via bond graphs [10], [11]. Constructing compact and reducedorder models from a complex large-scale $\mathrm{pH}$ system is a very active research area. However, all these methods require full knowledge about the model/process such as model parameters and discretization schemes. As discussed earlier, this may not be possible in several scenarios. Hence, we focus on $\mathrm{pH}$ modeling using only input-output data.

\footnotetext{
${ }^{1}$ All authors are affiliated with the Max Planck Institute for Dynamics of Complex Technical Systems, Sandtorstr. 1, 39106 Magdeburg, Germany. Email: \{cherifi,goyalp, benner\}@mpi-magdeburg.mpg.de.

${ }^{2}$ The author is also a professor in the Faculty of Mathematics at Otto von Guericke University, Universitätsplatz 2, 39106 Magdeburg, Germany.
}

With this aim, the authors in [12], [13] proposed realization methods based on frequency-domain measurements. In [12], the author presented a method that uses frequency response data to find a realization, not necessarily in a $\mathrm{pH}$ form, however, a linear matrix inequality (LMI) based approach was proposed to find an underlying $\mathrm{pH}$ realization. This extends the results presented in [14], [15]. On the other hand, the authors in [13] have extended the Loewner framework [16] that directly yields a $\mathrm{pH}$ model by the construction of the Loewner and shifted Loewner pencils in a particular way. However, the frequency response data of a system may not be readily available in some applications, see, e.g., [17]. In these types of applications, it might be easier to obtain timedomain experimental data or collect simulation data for a given input using proprietary software.

One example, where a non-intrusive approach of modeling $\mathrm{pH}$ systems using time-domain data would be of great importance, is the modeling of gas transport networks. Although there exists a general hierarchy of submodels, see [17], there is no general model available. In particular, the compressor stations in gas networks do not have a first-principles model, but time-domain input-output data can be obtained. Then, in order to express the whole gas transport system as a single $\mathrm{pH}$ model using a network of hierarchies of $\mathrm{pH}$ sub-models, a non-intrusive method is of high interest to generate a model for this component. Another example, in this direction, is the modeling of a cable-driven parallel robot [18]. Such models are used to design physics-shaping controllers. Although there have been attempts to analytically build $\mathrm{pH}$ models, the analysis in [18] shows the limitations and the complexity in order to derive analytically large and complex $\mathrm{pH}$ models. Similar motivational examples can be found, e.g., in power electronics [19] and continuous stirred tank reactors [20].

Having noted various applications, we now seek to realize an underlying $\mathrm{pH}$ model of a process using time-domain data. Since there exist tools to build $\mathrm{pH}$ models from the frequency response data of a system [13], our primary goal is to estimate the frequency response data using the time-domain data. For this, we first impose the linearity assumption on the underlying dynamics. Under this assumption, there exist techniques that allow us to achieve our desire goal, see, e.g., [21]. By combining the ideas discussed in [13] and [21], we propose a procedure to infer an underlying $\mathrm{pH}$ realization using time-domain data obtained, e.g., using proprietary software, or in an experimental set-up.

The rest of the paper is structured as follows. In the following section, we introduce the $\mathrm{pH}$ framework and present the state of the art methods to infer $\mathrm{pH}$ realizations from 
data. Subsequently, in Section III, we discuss a time-domain Loewner framework that allows us to infer an underlying $\mathrm{pH}-$ model using time-domain data. Section IV demonstrates the proposed procedure by means of two numerical examples. In Section V, we conclude the paper with a short summary.

\section{Port-Hamiltonian Systems And Previous Work}

The $\mathrm{pH}$ framework is powerful as it inherently encodes underlying physical principles directly into the structure of the system model. An LTI pH system can be written in the following form [15]:

$$
\begin{aligned}
& \dot{\mathbf{x}}(t)=(\mathbf{J}-\mathbf{R}) \mathbf{Q} \mathbf{x}(t)+(\mathbf{F}-\mathbf{P}) \mathbf{u}(t), \\
& \mathbf{y}(t)=(\mathbf{F}+\mathbf{P})^{\top} \mathbf{Q} \mathbf{x}(t)+(\mathbf{S}+\mathbf{N}) \mathbf{u}(t),
\end{aligned}
$$

where $\mathbf{J} \in \mathbb{R}^{n \times n}$ is a skew-symmetric matrix, $\mathbf{R} \in \mathbb{R}^{n \times n}$ is a positive semi-definite matrix, $\mathbf{F} \pm \mathbf{P} \in \mathbb{R}^{n \times m}$ are port matrices, $\mathbf{S}+\mathbf{N}$ is the feed-through from the input to the output with $\mathbf{S}=\mathbf{S}^{\top} \geq 0 \in \mathbb{R}^{m \times m}, \mathbf{N}=-\mathbf{N}^{\top} \in \mathbb{R}^{m \times m}$, and $\mathbf{Q} \in \mathbb{R}^{n \times n}$ is a positive semi-definite that is associated with the Hamiltonian, $\mathcal{H}(x)=\frac{1}{2} \mathbf{x}^{\top} \mathbf{Q} \mathbf{x}$. There is a close relationship between $\mathrm{pH}$ and passive systems, see, e.g., [15], [22]. To assure that an LTI system of the form (1) is passive, the following condition is also required:

$$
\left[\begin{array}{cc}
\mathbf{R} & \mathbf{P} \\
\mathbf{P}^{\top} & \mathbf{S}
\end{array}\right] \geq 0
$$

A model having the $\mathrm{pH}$ structure has many intrinsic spectral properties [23] - pH systems are robust under structured perturbations [24], [25], pH systems are closed under powerconserving interconnection [9], and model reduction of largescale $\mathrm{pH}$ systems via Galerkin projection yields low-order systems that preserve the $\mathrm{pH}$ structure, see, e.g., [26], [27], [28].

The main contributions, so far, in the direction of inferring $\mathrm{pH}$ realizations from data can be found in [12], [13], [29]. The methods in [12], [13] are based on frequency-domain data. The authors in [13] proposed a $\mathrm{pH}$ realization method from frequency-domain data based on the Loewner framework [16]. However, the method requires data at the spectral zeros in the spectral directions which may not be easily available. Therefore, the authors in [13] proposed to infer first a state-space model using the standard Loewner and then obtain samples at the spectral zeros in the spectral directions using the inferred state-space model. This approach is computationally efficient since an underlying $\mathrm{pH}$ system can be analytically determined. Recently, in [12], a datadriven method to learning $\mathrm{pH}$ systems based on a solution of the passivity LMI [15] is proposed. Moreover, In [29], the authors proposed approaches to realize port-Hamiltonian systems using three different approaches. The goal is to learn a $\mathrm{pH}$ system in the form (1) for given input-output timedomain data. In these methods, the idea is first to estimate frequency domain data as done in [21], followed by applying the Loewner approach to obtain a state-space model of the form:

$$
\begin{aligned}
& \dot{\mathbf{x}}(t)=\mathbf{A x}(t)+\mathbf{B u}(t), \quad \mathbf{x}(0)=0, \\
& \mathbf{y}(t)=\mathbf{C} \mathbf{x}(t)+\mathbf{D u}(t) .
\end{aligned}
$$

Then, given the system (2), the authors seek an underlying $\mathrm{pH}$ structure and discuss approaches by means of, e.g., the solution of an LMI or the Luré equations, or finding the nearest $\mathrm{pH}$ realization, belonging to the set of all admissible passive systems by solving the following optimization problem:

$$
\inf _{\mathbf{J}, \mathbf{R}, \mathbf{Q}, \mathbf{F}, \mathbf{P}, \mathbf{S}} \mathcal{G}(\mathbf{J}, \mathbf{R}, \mathbf{Q}, \mathbf{F}, \mathbf{P}, \mathbf{S})
$$

subject to $\mathbf{J}^{\top}=-\mathbf{J}, \mathbf{Q} \geq 0$ and $\left[\begin{array}{cc}\mathbf{R} & \mathbf{P} \\ \mathbf{P}^{\top} & \mathbf{S}\end{array}\right] \geq 0$, and where

$$
\begin{aligned}
\mathcal{G}(\mathbf{J}, \mathbf{R}, \mathbf{Q}, \mathbf{F}, \mathbf{P}, \mathbf{S}) & =\|\mathbf{A}-(\mathbf{J}-\mathbf{R}) \mathbf{Q}\|_{F}^{2}+\|\mathbf{B}-(\mathbf{F}-\mathbf{P})\|_{F}^{2} \\
& +\left\|\mathbf{C}-(\mathbf{F}+\mathbf{P})^{\top} \mathbf{Q}\right\|_{F}^{2}+\left\|\frac{\mathbf{D}+\mathbf{D}^{\top}}{2}-\mathbf{S}\right\|_{F}^{2},
\end{aligned}
$$

and $\mathbf{N}$ is set to $\mathbf{N}=\frac{\mathbf{D}-\mathbf{D}^{\top}}{2}$, see [30] for details. This method allows for a general and versatile realization procedure. However, solving the above optimization problem is a challenging task. Towards this, the authors propose an algorithm to solve this optimization problem that is based on a fast projected gradient method (FGM) [30]. The method is in general faster than the standard projected gradient method for this type of problems where the objective function is non-convex. This also requires a restarting procedure to ensure that the algorithm converges. In addition, Obtaining a suitable solution for this optimization problem requires choosing a good initial system. The LMI based initialization procedure proposed in [30] works well when the initial system is close to being passive. However, the algorithm may get stuck in a local minimum. Also, solving the LMI may be computationally expensive if the system is of large-scale. It is worth noting that only one representation of $\mathrm{pH}$ systems is considered. There may be a nearer $\mathrm{pH}$ system with another representation e.g. in $\mathrm{pH} \mathrm{DAE}$ form.

In the following, we discuss a procedure that involve first inferring frequency response data [21], [29]. Then, we directly compute the underlying $\mathrm{pH}$ system using the method proposed in [13].

\section{TIME DOMAIN LOEWNER PORT-HAMILTONIAN REALIZATION}

Our main objective is to realize a $\mathrm{pH}$ system from given time-domain input-output data which can either be obtained in an experimental set-up or using proprietary software. In essence, we first infer the frequency response data of the system using time-domain input-output data. This problem has been very well studied in the literature, see, e.g., [31], [32], [33], where frequency response data are typically inferred using the impulse response. However, in this paper, we follow the method presented in [21] to estimate the frequency data points at pre-defined interpolation points by designing an appropriate input. This is done by solving a least-squares problem. See [21] for details.

Let us assume that we excite the system using an input $\mathbf{u}(t)$ and consider $K$ samples of $\mathbf{u}(t)$ at the time $k T_{s}$, denoted as $\mathbf{u}_{k}:=\mathbf{u}\left(k T_{s}\right)$, where $T_{s}$ is the sampling time and $k \in\{0, \ldots, K\}$. Then, using the discrete Fourier transform, 
we can write

$$
\mathbf{u}_{k}=\sum_{i=0}^{K-1} \mathbf{U}_{i} \mathbf{q}_{i}^{k},
$$

where $\mathbf{U}_{i}$ are the corresponding (discrete) Fourier coefficients and $\mathbf{q}_{i}=e^{\frac{2 \pi \sqrt{-1}}{K} i}$ for $i \in\{0, \ldots, K-1\}, k \in$ $\{0, \ldots, K-1\}$. Moreover, under the linearity assumption, we can write the output sequence as follows:

$$
\mathbf{y}_{k}=\sum_{i \in \Gamma_{r}} \mathbf{U}_{i} \mathbf{H}_{k}\left(\mathbf{q}_{i}\right) \mathbf{q}_{i}^{k},
$$

where $\Gamma_{r}=\left\{i_{1}, \ldots, i_{r}\right\}$ are the indices of the $r$ non-zero Fourier coefficients $\left\{\mathbf{U}_{1}, \ldots, \mathbf{U}_{r}\right\}$. Equation (3) gives us a direct relationship between the output $\mathbf{y}_{k}$ and the approximate frequency response data $\mathbf{H}_{k}$ sampled at the frequency $\mathbf{q}_{i}$. To estimate the frequency response data of the system, one can solve a least-squares problem of the form:

$$
\widehat{\mathbf{H}}=\underset{\widehat{\mathbf{H}}_{1}^{\prime}, \ldots, \widehat{\mathbf{H}}_{r}^{\prime} \in \mathbb{C}}{\arg \min } \sum_{k=k_{\min }}^{K-1}\left(\mathbf{y}_{k}-\sum_{l=1}^{r} \mathbf{U}_{i_{l}} \widehat{\mathbf{H}}_{l}^{\prime} \mathbf{q}_{i_{l}}^{k}\right)^{2}
$$

with the solution

$$
\widehat{\mathbf{H}}=\left[\widehat{\mathbf{H}}_{1}, \ldots, \widehat{\mathbf{H}}_{r}\right]^{\top}
$$

and $k_{\min }$ is chosen such that (4) is overdetermined and has a unique solution. This is generally true when the system reaches a steady state. Equation (4) can be rewritten as

$$
\underset{\widehat{\mathbf{H}} \in \mathbb{C}^{r}}{\arg \min }\|\mathbf{F} \widehat{\mathbf{H}}-\overline{\mathbf{y}}\|_{2}^{2}
$$

where $\mathbf{F} \in \mathbb{C}^{\left(\mathbf{K}-k_{\min }\right) \times r}$ is as follows :

$$
\mathbf{F}=\left[\begin{array}{ccc}
\mathbf{U}_{i_{1}} \mathbf{q}_{i_{1}}^{k_{\min }} & \ldots & \mathbf{U}_{i_{m}} q_{i_{r}}^{k_{\min }} \\
\vdots & \ddots & \vdots \\
\mathbf{U}_{i_{1}} \mathbf{q}_{i_{1}}^{K-1} & \ldots & \mathbf{U}_{i_{m}} q_{i_{r}}^{K-1}
\end{array}\right]
$$

and $\overline{\mathbf{y}}=\left[\mathbf{y}_{k_{\min }}, \ldots, \mathbf{y}_{K-1}\right]^{\top}$. Once we estimate the frequency response data $\widehat{\mathbf{H}}_{1}, \ldots, \widehat{\mathbf{H}}_{r}$ at the corresponding interpolation points $\mathbf{q}_{i_{1}}, \ldots, \mathbf{q}_{i_{r}}$, the classical Loewner framework can be employed [16]. For this, the first step is to select an even number $\widehat{m}<=r$ of interpolation points and define $n:=\frac{\widehat{m}}{2}$. Then, the set of interpolation points is partitioned into left and right interpolation points: $\mathbf{q}_{i_{1}}, \ldots, \mathbf{q}_{i_{n}}$ and $\mathbf{q}_{i_{n+1}}, \ldots, \mathbf{q}_{i_{\overparen{m}}}$, respectively. Then, we can construct the Loewner and shifted Loewner matrices as follows:

$$
\begin{aligned}
\widehat{\mathbb{L}}= & {\left[\begin{array}{ccc}
\frac{\widehat{\mathbf{H}}_{1}-\widehat{\mathbf{H}}_{n+1}}{\mathbf{q}_{i_{1}}-\mathbf{q}_{i_{n+1}}} & \cdots & \frac{\widehat{\mathbf{H}}_{1}-\widehat{\mathbf{H}}_{2 n}}{\mathbf{q}_{i_{1}}-\mathbf{q}_{i_{2 n}}} \\
\vdots & \ddots & \vdots \\
\widehat{\mathbf{H}}_{i_{n}}-\widehat{\mathbf{H}}_{i_{2 n}} & \ldots & \frac{\widehat{\mathbf{H}}_{i_{n}}-\widehat{\mathbf{H}}_{i_{2 n}}}{\mathbf{q}_{i_{n}}-\mathbf{q}_{i_{n+1}}}
\end{array}\right], } \\
\widehat{\mathbb{L}}_{s}= & {\left[\begin{array}{ccc}
\frac{\mathbf{q}_{i_{1}} \widehat{\mathbf{H}}_{1}-\mathbf{q}_{i_{n+1}}}{\mathbf{q}_{i_{1}}-\mathbf{q}_{i_{n+1}}} & \widehat{\mathbf{H}}_{n+1} \\
\vdots & \ldots & \frac{\mathbf{q}_{i_{1}} \widehat{\mathbf{H}}_{1}-\mathbf{q}_{i_{2 n}} \widehat{\mathbf{H}}_{2 n}}{\mathbf{q}_{i_{1}}-\mathbf{q}_{i_{2 n}}} \\
\frac{\mathbf{q}_{i_{n}} \widehat{\mathbf{H}}_{i_{n}}-\mathbf{q}_{i_{n+1}} \widehat{\mathbf{H}}_{i_{2 n}}}{\mathbf{q}_{i_{n}}-\mathbf{q}_{i_{n+1}}} & \cdots & \frac{\mathbf{q}_{i_{n}} \widehat{\mathbf{H}}_{i_{n}}-\mathbf{q}_{i_{2 n}} \widehat{\mathbf{H}}_{i_{2 n}}}{\mathbf{q}_{i_{n}}-\mathbf{q}_{i_{2 n}}}
\end{array}\right] . }
\end{aligned}
$$

This allows us to infer a discrete-time model in a generalized state-space form:

$$
\begin{aligned}
\widehat{\mathbf{E}} \mathbf{x}_{k+1} & =\widehat{\mathbf{A}} \mathbf{x}_{k}+\widehat{\mathbf{B}} \mathbf{u}_{k}, \\
\mathbf{y}_{k} & =\widehat{\mathbf{C}} \mathbf{x}_{k},
\end{aligned}
$$

where $\widehat{\mathbf{E}}=-\widehat{\mathbb{L}}, \widehat{\mathbf{A}}=-\widehat{\mathbb{L}}_{s}, \widehat{\mathbf{B}}=\left[\widehat{\mathbf{H}}_{1}, \ldots, \widehat{\mathbf{H}}_{n}\right]^{\top}, \widehat{\mathbf{C}}=$ $\left[\widehat{\mathbf{H}}_{n+1}, \ldots, \widehat{\mathbf{H}}_{2 n}\right]$ and it is assumed that the pencil $\left(s \widehat{\mathbb{L}}, \widehat{\mathbb{L}}_{s}\right)$ is regular. If it is not regular, then there exists a lowerorder model that interpolates the data which can be obtained by performing a compression step, see [16] for a detailed discussion.

The choice of the input $\mathbf{u}(t)$, the interpolation points and the number of samples $K$ should be chosen wisely so that the frequency response data can be estimated up to a satisfactory tolerance in the desired range. Typically, the range of the possible frequencies that can be chosen to estimate the data is:

$$
\left[\frac{2 \pi}{K}, \frac{2 \pi(K-1)}{K}\right]
$$

It shows that as the number of samples $K$ increases, the range of possible frequency increases. Moreover, the input should also be carefully chosen to span the frequency range of interest and so that it has non-zero Fourier coefficients only for the frequencies corresponding to the pre-defined interpolation points. This also ensures that the matrix $\mathbf{F}$ in (5) has low dimensions because the input is sparse in the Fourierdomain. To this purpose, the input is generally chosen to be a sum of cosine and sine signals:

$$
\mathbf{u}_{k}=\frac{1}{K} \sum_{l=1}^{m}(1+\jmath)\left(\cos \left(\frac{2 \pi i_{l} k}{K}\right)+\jmath \sin \left(\frac{2 \pi i_{l} k}{K}\right)\right),
$$

where $k \in\{0, \ldots, K-1\}$ and $i_{l}$ are the pre-defined indices of the non-zero Fourier coefficients. Note that the resulting system (6) is a discrete-time system since it is inferred using discrete sampling of input and output. However, a discrete-time system can be transformed into a continuoustime system based on the implicit Euler method where the discrete time frequency domain variable $\mathrm{z}$ is tranfomed to the contimous time frequency domain variable $s$ with the relation: $z=\frac{1}{1-s T_{s}}$. The continuous time system is then computed as:

$$
\mathbf{E}_{c}=\widehat{\mathbf{E}}, \quad \mathbf{A}_{c}=\frac{1}{T_{s}}(\widehat{\mathbf{A}}-\widehat{\mathbf{E}}), \quad \mathbf{B}_{c}=\frac{1}{T_{s}} \widehat{\mathbf{B}}, \mathbf{C}_{c}=\widehat{\mathbf{C}} .
$$

Once we have a realization of the system, we can construct a $\mathrm{pH}$ realization as discussed in [13]. In the following, we first define the spectral zeros and zero directions.

Definition 3.1: Given a transfer function $\mathbf{H}(s)$ of an order $n$ system, the pairs $\left(s_{j}, r_{j}\right), j \in\{1, \ldots, n\}$, are spectral zeros and zero directions if

$$
\Phi\left(s_{j}\right) r_{j}=0,
$$

where $\Phi(s):=\mathbf{H}^{*}(-s)+\mathbf{H}(s)$ and " $*$ " denotes the conjugate transpose. For a general state space representation, 
these spectral zeros and spectral directions can be computed by solving the generalized eigenvalue problem [34], [35]:

$$
\left[\begin{array}{ccc}
0 & \mathbf{A} & \mathbf{B} \\
\mathbf{A}^{T} & 0 & \mathbf{C}^{T} \\
\mathbf{B}^{T} & \mathbf{C} & \mathbf{D}+\mathbf{D}^{T}
\end{array}\right]\left[\begin{array}{c}
p_{j} \\
q_{j} \\
r_{j}
\end{array}\right]=s_{j}\left[\begin{array}{ccc}
0 & \mathbf{E} & 0 \\
-\mathbf{E}^{T} & 0 & 0 \\
0 & 0 & 0
\end{array}\right]\left[\begin{array}{c}
p_{j} \\
q_{j} \\
r_{j}
\end{array}\right] .
$$

Now, let us consider data as follows:

$$
\mathbf{H}\left(\lambda_{j}\right) r_{j}=w_{j}, \quad j \in\{1, \ldots, n\},
$$

where $\lambda_{j} \in \mathbb{C}^{+}, j \in\{1, \ldots, n\}$, are the spectral zeros of $\mathbf{H}(s)$ in the open right half-plane and $r_{j}$ are the corresponding zero directions. By making use of (8), we also have

$$
r_{j}^{*} \mathbf{H}\left(-\lambda_{j}^{*}\right)=w_{j}^{*}, \quad j \in\{1, \ldots, n\} .
$$

Next, we define the right and left tangential interpolations set as $\left(\lambda_{j}, r_{j}, w_{j}\right)$ and $\left(-\lambda_{j}^{*}, r_{j}^{*}, w_{j}^{*}\right)$, respectively. Then, using these interpolation conditions, we can obtain the Loewner and shifted Loewner matrices as follows:

$$
\begin{aligned}
& \mathbb{L}= {\left[\begin{array}{ccc}
\frac{r_{1}^{*} w_{1}+w_{1}^{*} r_{1}}{\lambda_{1}+\lambda_{1}^{*}} & \cdots & \frac{r_{1}^{*} w_{n}+w_{1}^{*} r_{n}}{\lambda_{n}+\lambda_{1}^{*}} \\
\vdots & \ddots & \vdots \\
\frac{r_{n}^{*} w_{1}+w_{n}^{*} r_{1}}{\lambda_{1}+\lambda_{n}^{*}} & \cdots & \frac{r_{n}^{*} w_{n}+w_{n}^{*} r_{n}}{\lambda_{n}+\lambda_{n}^{*}}
\end{array}\right], } \\
& \mathbb{L}_{s}=\left[\begin{array}{ccc}
\frac{\lambda_{1} r_{1}^{*} w_{1}+\lambda_{1}^{*} w_{1}^{*} r_{1}}{\lambda_{1}+\lambda_{1}^{*}} & \ldots & \frac{\lambda_{n} r_{1}^{*} w_{n}+\lambda_{1}^{*} w_{1}^{*} r_{n}}{\lambda_{n}+\lambda_{1}^{*}} \\
\vdots & \ddots & \vdots \\
\frac{\lambda_{1} r_{n}^{*} w_{1}+\lambda_{n}^{*} w_{n}^{*} r_{1}}{\lambda_{1}+\lambda_{n}^{*}} & \cdots & \frac{\lambda_{n} r_{n}^{*} w_{n}+\lambda_{n}^{*} w_{n}^{*} r_{n}}{\lambda_{n}+\lambda_{n}^{*}}
\end{array}\right] .
\end{aligned}
$$

This allows us to construct a state-space realization that also matches the transfer function at infinity as follows:

$$
\begin{aligned}
& \mathbf{E}=-\mathbb{L}, \quad \mathbf{A}=\mathbb{L}_{s}-\mathbf{R}^{*} \mathbf{D R}, \quad \mathbf{B}=-\mathbf{W}^{*}-\mathbf{R}^{*} \mathbf{D}, \\
& \mathbf{C}=-\mathbf{W}+\mathbf{D R}, \quad \mathbf{D}=\mathbf{D} .
\end{aligned}
$$

It is possible that the realization $(10)$ is complex. However, there exists an orthogonal transformation, allowing us to write the realization [10, as a real system, see [36]. Moreover, the system 10 can be transformed to the $\mathrm{pH}$ form as shown in (1) by a similarity transformation and satisfies all the necessary properties for a $\mathrm{pH}$ system. For a more detailed discussion, we refer to [13].

Remark 1: The direct feed-through term $\mathbf{D}$ can be estimated by observing the behavior at high frequencies. Moreover, it can also be assessed using the step response of the system. The step response near the time $t=0$ corresponds to the direct feed-through.

Remark 2: We note that we estimate the transfer function using the input-output data in a certain time interval. The accuracy of the estimation also depends on the sampling time. Precisely, if the sampling time is small, we can estimate the transfer function more accurately.

Remark 3: We would like to stress that there are some scenarios, where it is possible to have an explicit handle on the transfer function, thus one can generate frequency response data. In the case where generating data is expensive, we can adopt an adaptive scheme such as TF-IRKA [37] that allows us to choose wisely these frequency points (in $\mathcal{H}_{2}$-optimal sense) to evaluate the transfer function handle. Once we obtain a model from TF-IRKA, we can do the postprocessing to have an underlying $\mathrm{pH}$ model by generating the corresponding spectral zeros and directions data.

\section{NUMERICAL EXPERIMENTS}

In this section, we illustrate the proposed method to infer a linear $\mathrm{pH}$ realization from time-domain data by means of two examples. The input $u_{k}$ and the output $y_{k}$ of the system are measured with respect to time with a sampling time of $T_{s}=0.1 s$. and recorded as vectors $\mathbf{u}$ and $\mathbf{y}$ with length $K$ which corresponds to the number of samples taken. In the following two examples, we take $K=1000$ measurements. As discussed in Section III], the input is chosen to be a sum of sine and cosine functions of the form:

$$
\mathbf{u}_{k}=\frac{1}{K} \sum_{l=1}^{m}(1+\jmath)\left(\cos \left(\frac{2 \pi i_{l} k}{K}\right)+\jmath \sin \left(\frac{2 \pi i_{l} k}{K}\right)\right),
$$

where $k \in\{0, \ldots, K-1\}$, and $k_{\min }$ is set to $k_{\min }=\frac{1}{4} K$ such that to ensure that the outputs have entered a steady state after $k_{\min }[21]$.

\section{A. Oseen equations}

Consider the incompressible flow model of the Oseen equations, described in [38], [39], [40], [41]:

$$
\begin{array}{rlrl}
\partial_{t} v & =-(a . \nabla) v+\mu \Delta v-\nabla p+f, & & \text { in } \Omega \times(0, T], \\
0 & =-\operatorname{div} v, & \text { in } \Omega \times(0, T], \\
v & =0, & \text { on } \partial \Omega \times(0, T], \\
v & =v^{0}, & \text { in } \Omega \times 0,
\end{array}
$$

where $v$ and $p$ are the velocity and pressure variables, $a$ is the driving velocity, $\mu>0$ is the dynamic viscosity, and $\Omega=(0,1)^{2}$ with boundary $\partial \Omega$. Moreover, we assume that the forcing term $f$ is separable, i.e., $f(x, t)=b(x) u(t)$. Using a finite-difference discretization scheme and setting the dynamic viscosity to $\mu=1$ and $a \equiv\left[\begin{array}{ll}1 & 1\end{array}\right]$, we obtain a model of order 279. Notice that the discretized system is a descriptor $\mathrm{pH}$ system, however, it is important to note that the transfer function of the system is strictly proper. We aim at learning the underlaying $\mathrm{pH}$ system directly from the timedomain data obtained using the simulation of the model.

In order to estimate the transfer function, we collect the input-output data at the time interval $T_{s}=0.1 \mathrm{~s}$. Then, using the input-output data, we select $m=200$ interpolation points. The first $m / 2$ interpolation points $\left\{q_{1}, \ldots, q_{m / 2}\right\} \in$ $\left\{q_{0}, \ldots, q_{K-1}\right\}$ are selected as logarithmically equidistant frequencies, and the other $m / 2$ interpolation points such that the set is closed under complex conjugation. The system is truncated and converted back to a continuous time system based on backward euler method. The resulting order of the continuous time system after truncation is $n=8$. Once we have a continuous time system that is a minimal and $\mathrm{pH}$, we can determine the port-Hamiltonian realization as described in Section III The quality of the obtained $\mathrm{pH}$ realization is then compared with the original Oseen equation using Bode 

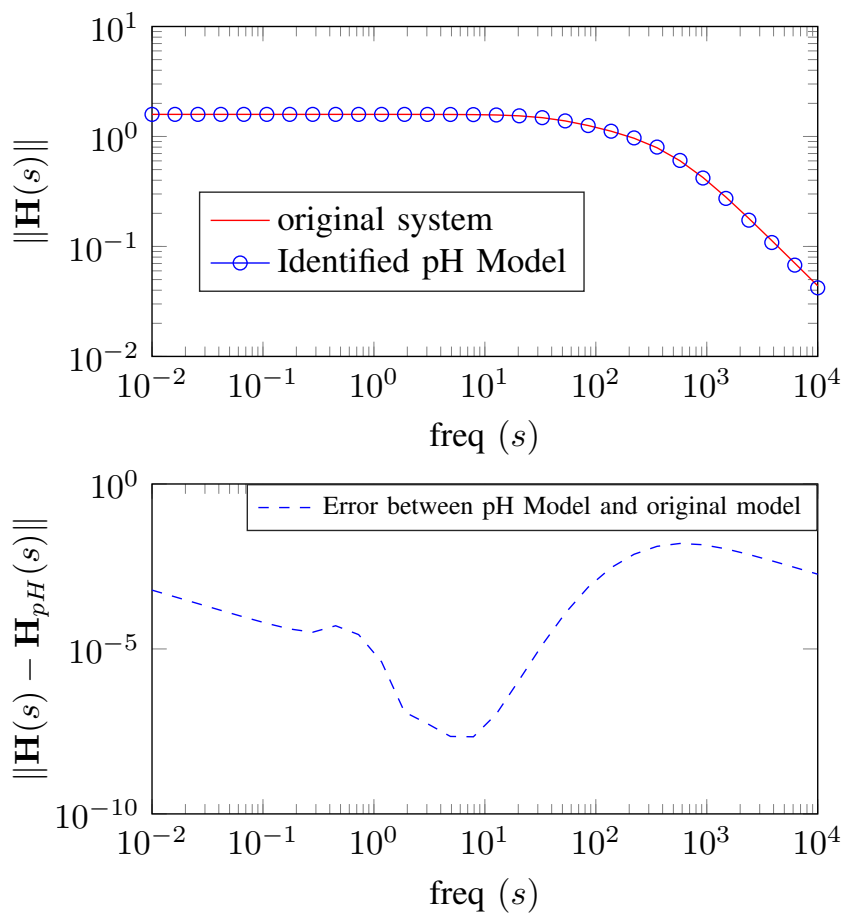

Fig. 1. Oseen equations: A comparison for the Bode plot of the original and the realized $\mathrm{pH}$ systems.

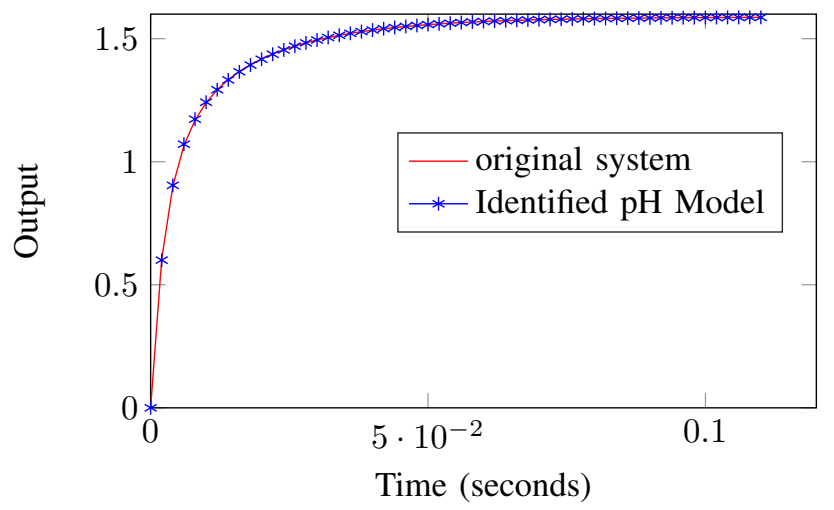

Fig. 2. Oseen equations: A comparison between the step response of the original and the realized $\mathrm{pH}$ systems.

plots which are shown in Figure 1. The figures show that we can obtain an underlying low-order $\mathrm{pH}$ system of the Oseen equations using time-domain input-output data.

We also also consider a comparison of the time domain response of the original system and the resulting $\mathrm{pH}$ formulation. Figure 2 shows a comparison between the step responses of the original and the resulting $\mathrm{pH}$ model.

It is important point that we estimate the transfer function using the input and output data at a certain time interval. The accuracy of the estimation depends on that sampling time. Precisely, if the sampling time is small, we can estimate the transfer function more accurately. Furthermore, the number of samples also has an influence on the estimation, i.e., more the samples better the estimation of the transfer function. Last but not least, the design of the input should be such

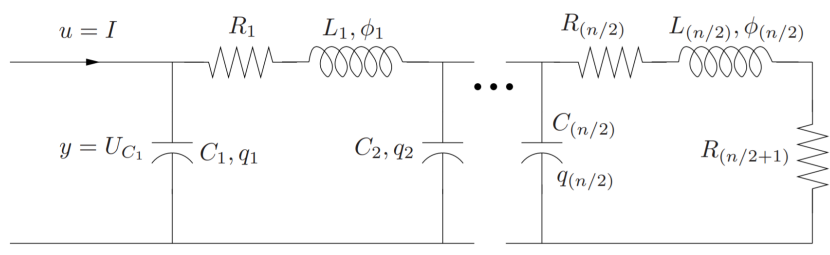

Fig. 3. RLC ladder circuit [42].
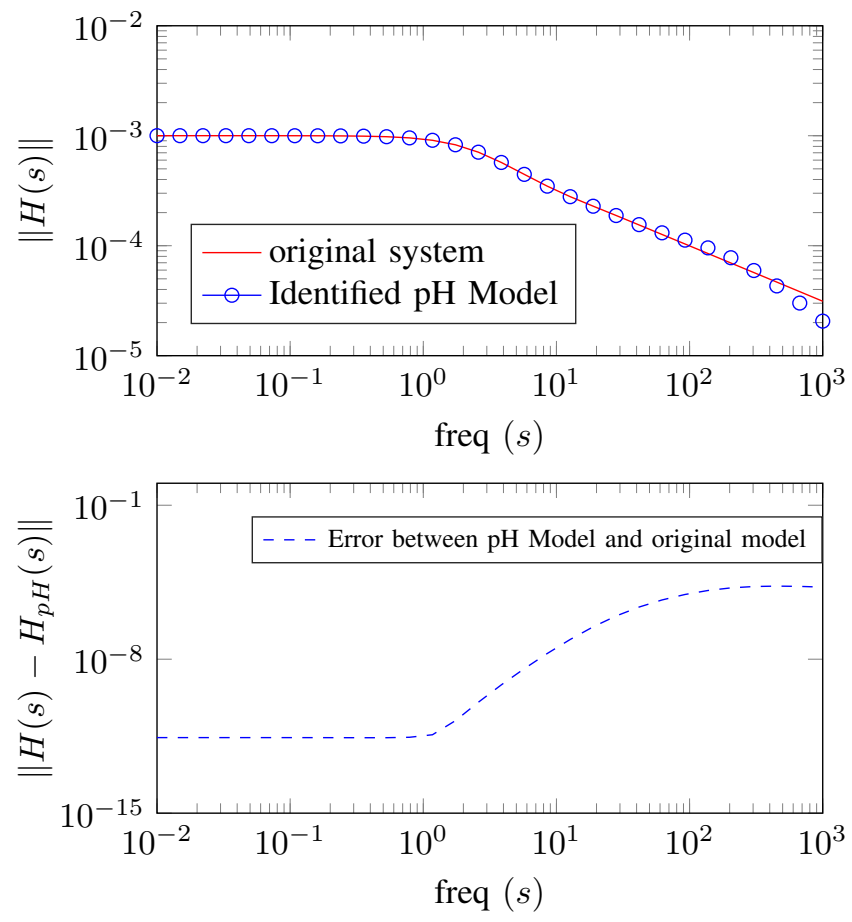

Fig. 4. RLC circuit: A comparison for the Bode plot of the original and the realized $\mathrm{pH}$ systems.

that it captures a wide range of frequencies or at least the the range of frequencies of interest so that we have a good estimate of the transfer function in that range.

\section{B. RLC circuit}

In the second example, we consider an RLC ladder circuit as shown in Figure 3. The current $I$ is taken as the input and the voltage $U_{c 1}$ over the first capacitor $C_{1}$ as the portHamiltonian output. This example is further discussed in [42]. The order of the system is 2000. In this case, we take a sampling time of $T_{s}=1 \mathrm{~s}$ and $m=100$ interpolation points. By keeping the same values for all other parameters parameters and by following the same steps as in the previous example, we obtain a $\mathrm{pH}$ realization of minimal order 3 . A comparison between Bode plots of the ladder network model and the realized $\mathrm{pH}$ system from the input-output data are shown in Figure 4 The figure again shows that we can realize the system in the $\mathrm{pH}$ form by using input-output data. The resulting system has also a low order compared to the original system where the data was taken. As in the previous example a comparison between the step response 


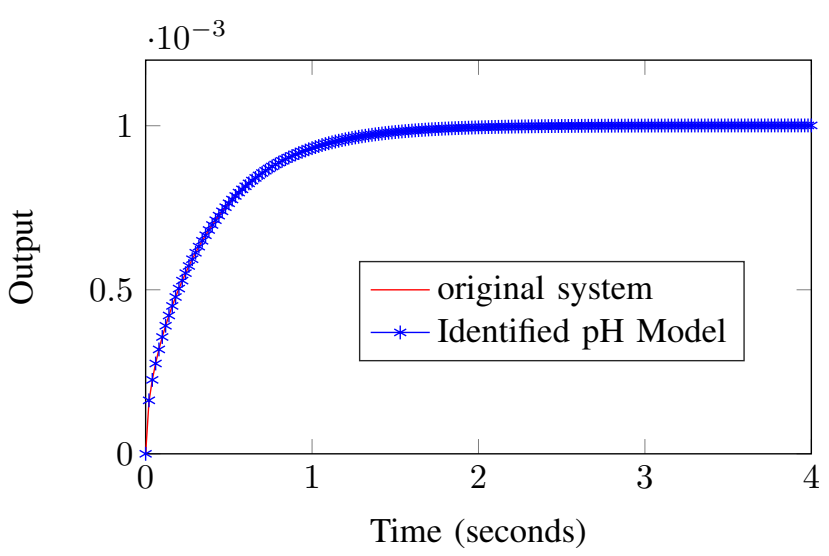

Fig. 5. RLC circuit: A comparison for the step response of the original and the realized $\mathrm{pH}$ systems.

of the original and the realized $\mathrm{pH}$ formulation is presented in Figure 5 .

\section{CONCLUSIONS}

In this paper, we have discussed a procedure to inferring a port-Hamiltonian realization using time-domain input-output data. For this, we have first estimated the frequency response data using time-domain input-output measurements. Using the frequency response data, we have then learned an underlying port-Hamiltonian realization by using the methodology proposed in [13]. We have illustrated the proposed procedure using two numerical examples. As further research, one can consider the extension of this work to multi-input multioutput (MIMO) systems and its real-world application, e.g., in gas networks.

\section{CODE AVAILABILITY}

The MATLAB code to reproduce the results presented in this paper can be found at https://gitlab.mpimagdeburg.mpg.de/cherifi/ph-realizations-from-timedomain-data .

\section{ACKNOWLEDGMENT}

P. Benner acknowledges support by the DFG Research Training Group RTG 2297/1 - MathCoRe at Magdeburg.

\section{REFERENCES}

[1] S. L. Brunton and J. N. Kutz. Data-Driven Science and Engineering: Machine Learning, Dynamical Systems, and Control. Cambridge University Press, 2019.

[2] J. Yu, C. Yan, and M. Guo. Non-intrusive reduced-order modeling for fluid problems: A brief review. Proc. the Institution of Mechanical Engineers, Part G: J. Aerospace Eng., 233(16):5896-5912, 2019.

[3] M. Guo and J.S. Hesthaven. Data-driven reduced order modeling for time-dependent problems. Comp. Meth. Appl. Mech. Eng., 345:75-99, 2019.

[4] B. Peherstorfer and K. Willcox. Data-driven operator inference for non-intrusive projection-based model reduction. Comp. Meth. Appl. Mech. Eng., 306:196-215, 2016.

[5] A. J. van der Schaft. Port-Hamiltonian systems: An introductory survey. In J. L. Verona M. Sanz-Sole and J. Verdura, editors, Proc. of the International Congress of Mathematicians, vol. III, Invited Lectures, pages 1339-1365, Madrid, Spain, 2006.
[6] B. Jacob and H. Zwart. Linear port-Hamiltonian systems on infinitedimensional spaces. Operator Theory: Advances and Applications, 223. Birkhäuser/Springer Basel AG, Basel CH, 2012.

[7] R. Ortega, A. J. van der Schaft, Y. Mareels, and B. M. Maschke. Putting energy back in control. Control Syst. Mag., 21:18-33, 2001.

[8] A. J. van der Schaft. Port-Hamiltonian systems: Network modeling and control of nonlinear physical systems. In Advanced Dynamics and Control of Structures and Machines, CISM Courses and Lectures, Vol. 444. Springer Verlag, New York, N.Y., 2004.

[9] A. J. van der Schaft and D. Jeltsema. Port-Hamiltonian systems theory: An introductory overview. Foundations and Trends in Systems and Control, 1(2-3):173-378, 2014.

[10] G. Golo, A. J. van der Schaft, P. C. Breedveld, and B. M. Maschke. Hamiltonian formulation of bond graphs. In A. Rantzer R. Johansson, editor, Nonlinear and Hybrid Systems in Automotive Control, pages 351-372. Springer, Heidelberg, 2003.

[11] P. C. Breedveld. Modeling and Simulation of Dynamic Systems using Bond Graphs, pages 128-173. EOLSS Publishers Co. Ltd./UNESCO, Oxford, UK, 2008.

[12] C. Beattie. Data-driven modeling and optimization of dissipative dynamics. Workshop on Mathematics of Reduced Order Models, ICERM, Providence, RI, USA, 2020.

[13] P. Benner, P. Goyal, and P. Van Dooren. Identification of portHamiltonian systems from frequency response data. Technical report, 2019. arXiv: 1911.00080.

[14] C. Beattie, V. Mehrmann, and H. Xu. Port-Hamiltonian realizations of linear time invariant systems. Preprint 23-2015, Institut für Mathematik, TU Berlin, 2015.

[15] C. Beattie, V. Mehrmann, and P. Van Dooren. Robust port-Hamiltonian representations of passive systems. Automatica, 100:182-186, 2019.

[16] A. J. Mayo and A. C. Antoulas. A framework for the solution of the generalized realization problem. Linear Algebra Appl., 425(2-3):634662, 2007.

[17] P. Domschke, B. Hiller, J. Lang, and C. Tischendorf. Modellierung von Gasnetzwerken: Eine Übersicht. Preprint 2717, Fachbereich Mathematik, TU Darmstadt, 2017.

[18] C. Schenk, B. Yüksel, C. Secchi, and H.H. Bülthoff. Port Hamiltonian modeling of a cable driven robot. IFAC-PapersOnLine, 51(3):161-168, 2018.

[19] M. Cupelli, S. K. Gurumurthy, S. K. Bhanderi, Y. Zhiqing, P. Joebges, A. Monti, and R. W. De Doncker. Port controlled Hamiltonian modelling and IDA-PBC control of dual active bridge converters for dc microgrids. IEEE Trans. Industrial Electronics, 2019.

[20] H. Hoang, F. Couenne, C. Jallut, and Y. Le Gorrec. The port Hamiltonian approach to modeling and control of continuous stirred tank reactors. Journal of Process Control, 21(10):1449-1458, 2011.

[21] B. Peherstorfer, S. Gugercin, and K. Willcox. Data-driven reduced model construction with time-domain Loewner models. SIAM J. Sci. Comput., 39:A2152-A2178, 2017.

[22] C. I. Byrnes, A. Isidori, and J. C. Willems. Passivity, feedback equivalence, and the global stabilization of minimum phase nonlinear systems. IEEE Trans. Autom. Control, 36:1228-1240, 1991.

[23] C. Mehl, V. Mehrmann, and M. Wojtylak. Linear algebra properties of dissipative Hamiltonian descriptor systems. SIAM J. Matrix Anal. Appl., 39:1489-1519, 2018.

[24] C. Mehl, V. Mehrmann, and P. Sharma. Stability radii for linear Hamiltonian systems with dissipation under structure-preserving perturbations. SIAM J. Matrix Anal. Appl., 37(4):1625-1654, 2016.

[25] C. Mehl, V. Mehrmann, and P. Sharma. Stability radii for real linear Hamiltonian systems with perturbed dissipation. Bit Numerical Mathematics, 57:811-843, 2017.

[26] C. Beattie and S. Gugercin. Structure-preserving model reduction for nonlinear port-Hamiltonian systems. In 50th IEEE Conference on Decision and Control and European Control Conference (CDC-ECC), 2011, pages 6564-6569. IEEE, 2011.

[27] S. Gugercin, R. V. Polyuga, C. Beattie, and A. J. van der Schaft. Structure-preserving tangential interpolation for model reduction of port-Hamiltonian systems. Automatica, 48:1963-1974, 2012.

[28] R. V. Polyuga and A. J. van der Schaft. Structure preserving model reduction of port-Hamiltonian systems by moment matching at infinity. Automatica, 46:665-672, 2010.

[29] K. Cherifi, V. Mehrmann, and K. Hariche. Numerical methods to compute a minimal realization of a port-Hamiltonian system. Technical report, 2019. arXiv:1903.07042. 
[30] N. Gillis and P. Sharma. Finding the nearest positive-real system. SIAM J. Matrix Anal. Appl., 56(2):1022-1047, 2018.

[31] L. Ljung. System identification. Prentice Hall, 1987.

[32] A. C. Ionita and A. C. Antoulas. Matrix pencils in time and frequency domain system identification. Control, Robotics and Sensors. Institution of Engineering and Technology, pages 79-88, 2012.

[33] J. Hokanson. Numerically Stable and Statistically Efficient Algorithms for Large Scale Exponential Fitting. PhD thesis, Rice University, 2013.

[34] D. C. Sorensen. Passivity preserving model reduction via interpolation of spectral zeros. Syst. Control Lett., 54(4):347-360, 2005.

[35] P. Benner and H. Faßbender. Numerische Methoden zur passivitätserhaltenden Modellreduktion. at-Automatisierungstechnik, 54(4): 153-160, 2006.

[36] A. C. Antoulas, S. Lefteriu, and A.C. Ionita. A tutorial introduction to the Loewner framework for model reduction. In P. Benner, M. Ohlberger, A. Cohen, and K. Willcox, editors, Model Reduction and Approximation: Theory and Algorithms, Computational Science Engineering, pages 335-376. Society for Industrial and Applied Mathematics, SIAM, 2017.

[37] C. Beattie and S. Gugercin. Realization independent $\mathcal{H}_{2}$ approximation. In 51th IEEE Conference on Decision and control (CDC). IEEE, 2012

[38] M. Heinkenschloss, D. C. Sorensen, and K. Sun. Balanced truncation model reduction for a class of descriptor systems with application to the Oseen equations. SIAM J. Sci. Comput., 30(2):1038-1063, 2008.

[39] S. Hauschild, N. Marheineke, and V. Mehrmann. Model reduction techniques for linear constant coefficient port-Hamiltonian differentialalgebraic systems. 2019. arXiv:1901.10242.

[40] C. Beattie, S. Gugercin, and V. Mehrmann. Structure-preserving interpolatory model reduction for port-Hamiltonian differential-algebraic systems. 2019. arXiv:1910.05674.

[41] P. Benner, J. Saak, and M. M. Uddin. Balancing based model reduction for structured index-2 unstable descriptor systems with application to flow control. Numer. Algebra Control Optim., 6(1):1-20, March 2016.

[42] R. V. Polyuga. Model reduction of port-Hamiltonian systems. $\mathrm{PhD}$ thesis, University of Groningen, 2010. 